\title{
Análise do comportamento estratégico em preços no mercado de gasolina brasileiro: modelando volatilidade
}

Silvinha Pinto Vasconcelos"

Claudio Fóffano Vasconcelos"*

Resumo: Neste trabalho são apresentados modelos ARCH e GARCH que podem ser usados como uma metodologia complementar para filtrar acusações de cartel em mercados de gasolina a varejo. Para mostrar isto, foi feito um exercício usando dados semanais de preços de gasolina para São Paulo, Florianópolis e Recife para examinar a média e a variância destas séries temporais. Os resultados confirmam a hipótese de maiores preços durante o suposto período de conspiração em São Paulo e Recife. Porém, a hipótese de menor variância se confirmou somente em Recife. Finalmente, acreditamos que este trabalho contribuiu para a discussão de como detectar colusão tácita ou secreta porque recomendamos uma técnica econométrica que requer somente dados de preços médios e uma quantidade mínima de dados.

Palavras-chave: Paralelismo de Preços, Mercado de Gasolina, Antitruste.

Abstract: In this work it is presented $\mathrm{ARCH}$ and GARCH models that can be a complementary methodology to screen cartels complaints in retail gasoline markets. To show that, it was performed an exercise using weekly gasoline price data for São Paulo, Florianópolis and Recife to examine the mean and the variance of these times series. The results confirm the hypothesis of bigger prices during the supposed conspiracy period in São Paulo and Recife. Therefore, the hypothesis of a smaller variance was confirmed just only Recife. Finally, we believe that this work improved the discussion about how to detect tacit or secret collusion because we recommended an econometric technique which requires just data on average prices and a minimum amount of data.

Keywords: Price Parallelism, Gasoline Market, Antitrust.

JEL Classification: L41; L51; L95.

\footnotetext{
* Professora Adjunto do Departamento de Análise Econômica da Faculdade de Economia da UFJF. E-mail: silvinha.vasconcelos@ufjf.edu.br

** Professor Adjunto do Departamento de Economia e Finanças da Faculdade de Economia da UFJF. E-mail: claudio.foffano@ufjf.edu.br
} 


\section{Introdução}

Recentemente, em uma operação chamada "274", a Polícia Federal brasileira anunciou o desmantelamento de um cartel de gasolina que operava em João Pessoa e mantinha o preço alinhado em $R \$ 2,74$ (o que deu origem ao nome da operação). Este cartel foi sustentado pela ameaça de guerra predatória de preços contra os postos que tentassem competir com os que praticavam tal política, sendo que, na composição do conjunto de provas, a Polícia Federal se valeu de escutas telefônicas, como é de praxe. ${ }^{1}$ Que este fenômeno não é novo, a própria literatura indica bem, conforme salienta Grossman (2004): os empresários freqüentemente irão entrar em conluios. Mas a literatura indica, inclusive, que o problema da obtenção de provas de cartel ainda pode receber contribuições importantes da Teoria Econômica.

Tendo isto em vista, neste artigo apresentamos e discutimos uma ferramenta empírica que pode ser usada para analisar a existência de colusão em preços no mercado de gasolina a varejo. Uma pesquisa prévia foi feita baseada no mesmo mercado como estudo de caso, ${ }^{2}$ sendo que partiu-se do princípio de que os modelos de mercado sobre jogos não cooperativos repetidos infinitamente têm sido usados como uma indicação de como melhorar a metodologia de detecção de cartéis. No caso específico de paralelismo de preços, a regra legal é que conduta não é o bastante para provar acordo colusivo, pois, na terminologia de jogos, ela não provê a diferenciação entre equilíbrio de maximização conjunta de lucros e equilíbrio de Nash.

Seguindo esta regra, o objetivo central de Vasconcelos e Vasconcelos (2005) foi oferecer uma contribuição metodológica de modelos dinâmicos na tarefa de buscar provas de colusão secreta ou tácita, admitindo que paralelismo é somente uma evidência de infração à concorrência. Assim, os autores indicaram um método complementar de séries temporais que identifica uma relação causal e de longo prazo das variáveis estratégicas em um cartel.

No presente artigo, todavia, nós damos um passo adiante examinando outra metodologia de séries temporais: considerando as características dos preços de gasolina, modelaremos volatilidade. Sob esta abordagem, nossa análise ainda difere da metodologia oficial da Autoridade Antitruste brasileira que é usada para identificar em quais casos a investigação de cartel deve prosseguir. E esta nova abordagem

1 Para mais detalhes, ver http://www.mj.gov.br/noticias/2007/maio/RLS04052007cartel.htm

2 Ver Vasconcelos e Vasconcelos (2005). 
também refuta a apreciação de Ragazzo e Silva (2006), que descreve a metodologia oficial como mais eficiente e mais simples do que a de Vasconcelos e Vasconcelos (2005).

Escolhemos estudar mercados de gasolina porque eles frequentemente têm sido investigados pelas Autoridades Antitruste, dadas as freqüentes denúncias de acordos de cartel em preços no Brasil. ${ }^{3}$ $\mathrm{E}$, como os fundamentos teóricos deste assunto já foram extensivamente expostos, nós não iremos discutir esta base novamente. Basta lembrar que os estudos teóricos sobre paralelismo de preços predizem que as firmas que se comportam estrategicamente irão manter estratégias de preços que são voláteis porém similares. ${ }^{4}$

Outro comentário introdutório: a metodologia apresentada aqui pode ser classificada como nova porque a literatura brasileira sobre paralelismo de preços é ainda incipiente e somente poucos artigos estão disponíveis até então. ${ }^{5}$ Consequentemente, discutimos a metodologia complementar mencionada anteriormente (que não foi aplicada à análise do grau de competição no mercado de gasolina a varejo) à luz da pesquisa desenvolvida por Bolotova, Connor e Miller (2005).

A seção seguinte provê uma análise dos elementos da metodologia usada pela Autoridade Antitruste brasileira. A seção 3 discute o novo método e tem uma aplicação desta abordagem para o caso brasileiro. Os resultados estão na seção 4. Na seção 5 está a conclusão do artigo.

\section{0 critério da Autoridade Antitruste brasileira para avaliar denúncias de cartel no mercado de gasolina a varejo ${ }^{6}$}

No Brasil, a Secretaria de Acompanhamento Econômico desenvolveu uma metodologia para analisar as denúncias sobre o comportamento das firmas no mercado de gasolina a varejo levando em consideração preços e margens de lucro. Tal método é uma primeira tentativa de obter um filtro na tarefa de monitoramento dos cartéis e

\footnotetext{
3 Assim como em todo o mundo. Ver, por exemplo, Competition Bureau (1994); Slade (1987); Kovac, Putzová e Zemplinerová (2005); Ragazzo e Silva (2006).

4 Ver Baker (1993), Buccirossi (2002), Kovacic (1993), Macleod (1985), Normann (2000), Phlips (1998) e Yao e DeSanti (1993). Em Vasconcelos e Vasconcelos (2005) nós discutimos estas idéias em detalhes.

5 Ver Ragazzo e Silva (2006).

6 Esta seção resume a metodologia descrita em OECD (2006), mas também pode ser achada em Ragazzo and Silva (2006).
} 
ainda está sob discussão, resumindo-se em três elementos: a) a tendência da margem de lucro: se a margem de lucro decrescer, o mercado é considerado competitivo e a denúncia é rejeitada; b) a correlação linear: se não existe um link entre o aumento da margem com a redução da dispersão de preço (ou o coeficiente de variação dos preços de varejo) o caso é arquivado; c) se existir tal aumento na margem, verifica-se então se os comportamentos de dispersão de preço e margem seguem o mesmo padrão dentro da área geográfica do estado (considerando que os custos de monitoramento do cartel no estado seria muito alto). Se seguirem, o caso é arquivado. Então, dá-se andamento aos processos somente se um aumento da margem ligado à redução da dispersão do preço não seguir o mesmo padrão do estado. Nestes casos a investigação continua, com a busca de mais evidências via métodos investigativos permitidos pela legislação brasileira, tais como inspeções, escutas telefônicas, buscas e apreensões.

Todavia, um argumento central indica que este método não é inteiramente satisfatório como uma ferramenta de análise intertemporal de comportamento estratégico de fixação de preços: existe uma ambigüidade nas razões por detrás do comportamento que sustenta os cartéis. Por exemplo, se a margem de lucro decresce, como estar seguro que isto não é um período de punição depois de alguma firma ter trapaceado um acordo de cartel? Então, o decréscimo na margem de lucro não deve ser visto somente como um indicador de competição, pois as firmas podem estar punindo quem desviou. Em resumo, de um lado, o cartel pode resultar em um aumento na margem de lucro, e de outro, ele pode ter uma fase de punição com menores lucros, mas ainda isto será um comportamento anticompetitivo. E podemos dizer que este aspecto implica um maior limite na metodologia da autoridade antitruste se os dados forem restritos a um curto período de tempo.

Outro aspecto a salientar é o seguinte. Para séries com volatilidade, a variância não condicional pode ser constante até mesmo se a variância durante alguns períodos for inesperadamente grande. Inspeções casuais são arriscadas e testes formais são necessários para sustentar quaisquer impressões iniciais, mesmo que o forte padrão visual seja de heteroscedasticidade (variância inconstante) (ENDERS, 1995).

Isto posto, na próxima seção realizamos uma discussão acerca da nova metodologia que pode ser usada para complementar o procedimento oficial descrito anteriormente. Mas, primeiro, apresentamos um resumo do estudo que investiga o impacto da conduta colusiva no comportamento do preço de mercado usando uma técnica econométrica. 


\section{Uma nova proposta para filtrar denúncias de cartel}

\subsection{Complementando a revisão de literatura: o trabalho de Bolotova, Connor e Miller (2005)}

O artigo de Bolotova, Connor e Miller (2005) usa extensões de um modelo ARCH (autoregressive conditional heteroscedasticity) tradicional e um modelo $\mathrm{ARCH}$ generalizado $(\mathrm{GARCH})$ para examinar a diferença no comportamento de dois momentos da distribuição de preço durante colusão e na ausência dela usando preços de dois setores (produtores de ácido cítrico e lisina) que recentemente foram acusados de ação coordenada.

De acordo com os autores, existem algumas vantagens de usar estes modelos: primeiro, este procedimento pode ser usado na filtragem dos processos de investigação pela autoridade antitruste; segundo, ele pode ser também usado como uma alternativa a modelos econométricos comumente empregados nos procedimentos oficiais para quantificar 0 efeito da infração no preço de mercado; terceiro, os modelos $A R C H$ e GARCH requerem uma quantidade mínima de dados, no mínimo, séries temporais de preço para o produto do setor cartelizado antes, durante e depois do suposto ou conhecido período de infração.

Seguindo a literatura sobre cartéis, os autores formularam duas hipóteses. A primeira, relacionada ao comportamento do preço médio: eles esperavam que este fosse maior durante colusão do que sem colusão. A segunda se relaciona com o comportamento da variância: eles esperaram que esta fosse menor durante colusão do que sem colusão sob a suposição de uma colusão bem sucedida (i.e. quando a maior parte dos membros segue o preço estabelecido na maior parte do tempo e o cartel pode efetivamente detectar comportamento oportunístico de seus membros). ${ }^{7}$

Os autores encontraram fundamento para ambas as hipóteses no caso dos preços da lisina, mas para o ácido cítrico, os resultados fundamentam somente a hipótese do preço médio. Eles listaram duas explicações para este comportamento inesperado da variância: primeiro, o tempo de colusão para o mercado de ácido cítrico foi maior do que o tempo de colusão no mercado de lisina. A conseqüência de um período maior de tempo é a dificuldade de supervisionar e impor a disciplina interna do cartel. Segundo, um problema na disponibilidade dos dados pode ter tido um impacto nos resultados (havia

7 As hipóteses dos autores foram formuladas sob a suposição de que não houve mudança significativa no mercado onde o cartel operava que pudesse introduzir choques adicionais nos preços. 
menos dados disponíveis no caso do ácido cítrico). Com estas considerações iniciais, pode-se apresentar então o modelo propriamente dito, o que é feito a seguir.

\subsection{A técnica econométrica para selecionar denúncias de cartel}

\subsubsection{Especificação do modelo ${ }^{8}$}

Nesta seção nós discutimos os modelos $\operatorname{ARCH}(q)$ e GARCH $(p, q)$ usados na análise como uma proposta para a Autoridade Antitruste selecionar denúncias de cartel.

Engle apud Enders (1995, p.141) mostra que é possível modelar simultaneamente a média e a variância das séries. Considerando que a equação da média para uma variável observável Y no período $t$ segue, por exemplo, o processo autoregressivo de ordem um, denotado por AR(1),

$$
Y_{t}=a_{0}+a_{1} Y_{t-1}+u_{t}
$$

onde $u_{t}$ é um ruído branco,

$$
\begin{aligned}
& E\left(u_{t}\right)=0, E\left(u_{t}, u_{j}\right)=\left\{\begin{array}{l}
\sigma^{2} \text { for } j=0 \\
0 \text { for } j \neq 0
\end{array}\right. \\
& \text { e }\left|a_{1}\right|<1 .
\end{aligned}
$$

Por esta razão, a variância de $u_{t}$ é constante e igual à variância não condicional, $\sigma^{2}$. Agora suponha que a variância condicional não é constante. Uma estratégia simples para modelar a variância condicional seria estimar um processo $\operatorname{AR}(q)$ usando o quadrado do resíduo estimado da equação (1),

$$
\hat{u}_{t}^{2}=\alpha_{0}+\alpha_{1} \hat{u}_{t-1}^{2}+\alpha_{2} \hat{u}_{t-2}^{2}+\ldots+\alpha_{q} \hat{u}_{t-q}^{2}+v_{t}
$$

onde $v_{t}$ é um processo "ruído branco".

A expressão (2) implica que a projeção linear do erro ao quadrado da previsão de $\mathrm{Y}_{t}$ a partir dos prévios $q$ erros ao quadrado previstos é dada por

8 Nesta seção nós seguimos Enders (1995). 


$$
\hat{E}\left(\hat{u}_{t}^{2} \mid u_{t-1}^{2}, u_{t-2}^{2}, \ldots\right)=\alpha_{0}+\alpha_{1} u_{t-1}^{2}+\alpha_{2} u_{t-2}^{2}+\ldots+\alpha_{q} u_{t-q}^{2}
$$

Então, uma equação como a (2) é chamada de processo autoregressivo condicional heteroscedástico de ordem $q$, denotado por $u_{t} \sim \operatorname{ARCH}(q)$.

Uma representação mais parcimoniosa de um modelo $\mathrm{ARCH}$ (q) de mais alta ordem pode ser obtida usando o modelo GARCH( $p$, q) de Bollerslev. Bollerslev apud Enders (1995) estendeu o processo $\mathrm{ARCH}$ original de Engel ao desenvolver uma técnica que permite que a variância condicional seja um processo ARMA.

Suponha um processo $\mathrm{ARCH}(q)$ de $u_{t}$ caracterizado por

$$
u_{t}=v_{t} \sqrt{h_{t}}
$$

onde $\sigma_{\mathrm{v}}^{2}$ e 1

$$
E\left[u_{t}\right]^{2}=h_{t}=\alpha_{0}+\sum_{i=1}^{q} \alpha_{i} u_{t-i}^{2}+\sum_{j=1}^{p} \beta_{j} h_{t-j}
$$

Como $v_{t}$ é um processo ruído branco que é independente da realização passada de $u_{t-i}$, as médias condicional e não condicional de $u_{t}$ são iguais a zero. A questão é que a variância condicional de $u_{t}$ é dada por $E_{t-1} u_{t}^{2}=h_{t}$. Então, a variância condicional de $u_{t}$ é dada por $h$ na equação (4). Este modelo $\operatorname{ARCH}(p, q)$ generalizado, chamado $\operatorname{GARCH}(p, q)$, se aplica aos componentes autoregressivo e média móvel na variância heteroscedástica.

Para um processo $\operatorname{GARCH}(p, q)$ bem definido é necessário que $\alpha_{0}>0, \alpha_{i} \geq 0, \beta_{j} \geq 0$ e $\sum_{i=1}^{q} \alpha_{i}+\sum_{j=1}^{p} \beta_{j} \leq 1$

\subsubsection{Fonte, natureza dos dados e análise estatística descritiva}

Como dito anteriormente, nossa metodologia segue o trabalho de Bolotova, Connor e Miller (2005). Em outros termos, estimamos uma extensão dos modelos $\mathrm{ARCH}(q)$ e $\operatorname{GARCH}(p, q)$ tradicionais (introduzindo uma variável dummy referente ao período de conspiração) para analisar o comportamento da média e da variância do preço da gasolina a varejo.

Foram usados preços médios semanais de gasolina comum reportados pela ANP (Agência Nacional de Petróleo) (2006), para as cidades de Florianópolis, Recife e São Paulo, começando em janeiro e indo até dezembro de 2006. 
A figura 1 ilustra o comportamento dos preços de gasolina em São Paulo e a primeira impressão é de um padrão visual de flutuação significativa nos dados. A figura 2 ilustra o comportamento dos preços de gasolina em Florianópolis com dois momentos mostrando um decréscimo dos preços, o que pode ser pensado como um período de guerra os preços. Já a figura 3 retrata o comportamento dos preços em Recife. Os padrões visuais destas três séries temporais foram os indicativos para os períodos hipotéticos de cartel e as escolhas destes períodos foram feitas justamente com a intenção de esclarecer a metodologia proposta aqui.

Para o propósito deste estudo, assumimos que março de 2006 foi o início do período de conspiração e abril de 2006 foi o período final em São Paulo (de forma que a dummy assume valor um sobre este período). Para Florianópolis, assumimos que janeiro de 2006 foi o início do primeiro período de conspiração e março de 2006 foi o seu término; ainda supomos um segundo período de conspiração em Florianópolis, recomeçando em setembro de 2006, indo até dezembro de 2006 (de forma que a dummy assume o valor um sobre estes dois períodos separados). Para Recife o período de conspiração assumido foi de abril a julho de 2006.

Figura 1. Comportamento dos preços de gasolina, cidade de São Paulo, 2006

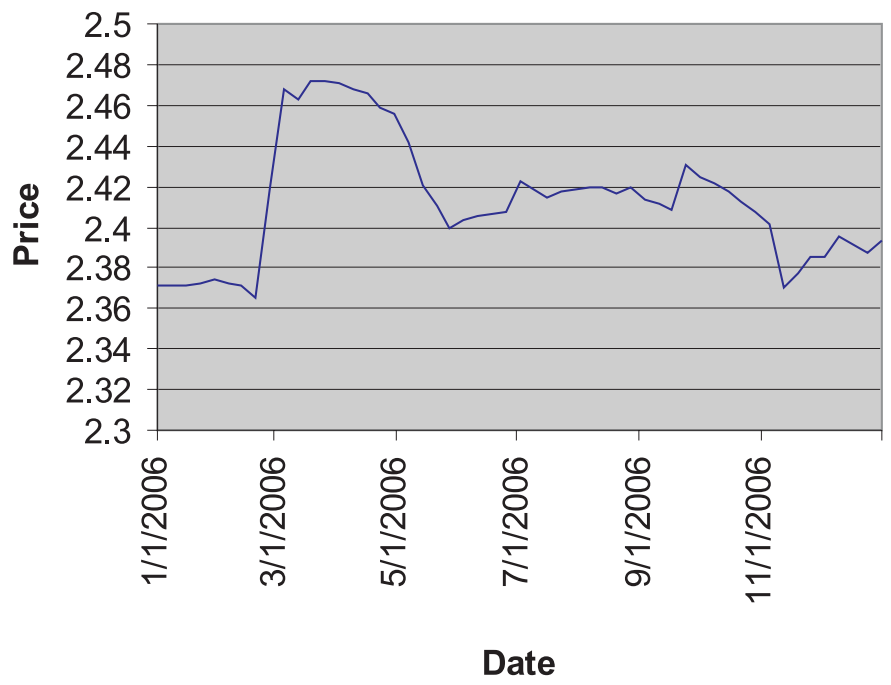

Fonte: ANP (2006). 
Figura 2. Comportamento dos preços de gasolina, cidade de Florianópolis, 2006

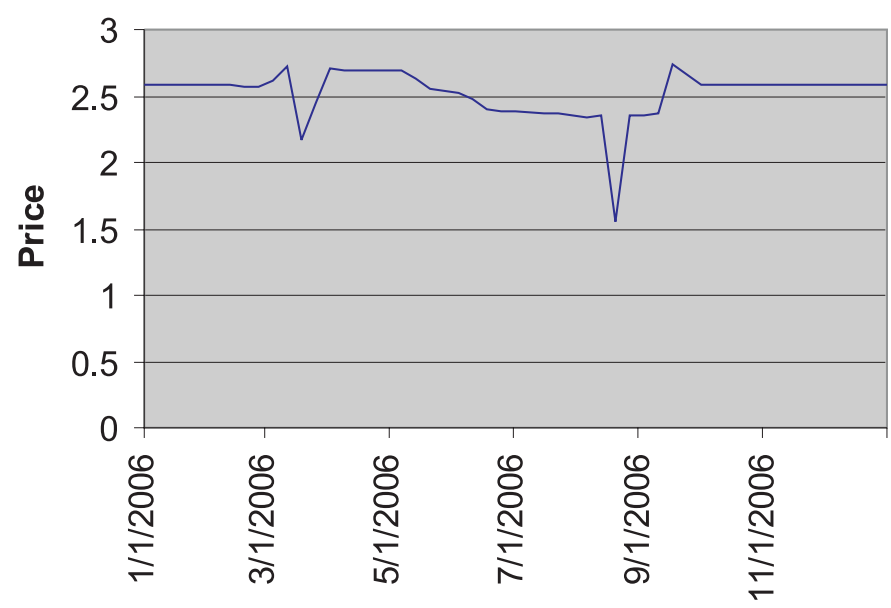

\section{Date}

Fonte: ANP (2006).

Figura 3. Comportamento dos preços de gasolina, cidade de Recife, 2006.

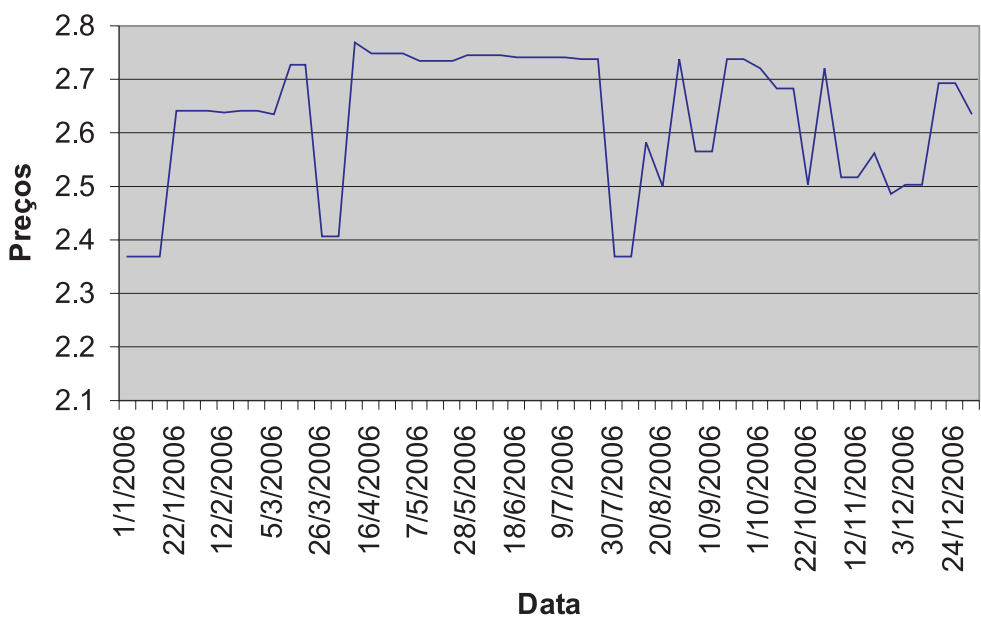

Fonte: ANP (2006).

A análise descritiva pode revelar alguma evidência da presença de comportamento colusivo nos três mercados de gasolina. Em São Paulo, a média do preço do suposto cartel foi $\mathrm{R} \$ 2,466$, maior do que 
a média do preço pré cartel $(\mathrm{R} \$ 2,376)$ e a média do preço pós cartel $(\mathrm{R} \$ 2,408)$. Além disso, a variância durante o período hipotético de cartel foi menor do que as variâncias dos preços nos períodos pré e pós cartel $(0,00003,0,000261$ e 0,000246, respectivamente).

Em Florianópolis, o preço médio do cartel nos primeiro e segundo períodos foram $R \$ 2,59$ e $R \$ 2,60$ respectivamente, maiores do que os preços do suposto período de guerra de preços $(R \$ 2,45)$. $\mathrm{E}$ as variâncias durante os períodos hipotéticos de cartel foram menores do que a variância do período de guerra de preço $(0,001707$; 0,001617; e 0,057208, respectivamente).

Já em Recife, a média do preço do suposto cartel foi $\mathrm{R} \$ 2,743,{ }^{9}$ maior do que a média do preço pré-cartel $(\mathrm{R} \$ 2,560)$ e a média do preço pós cartel $(R \$ 2,590)$. Além disso, a variância durante o período hipotético de cartel foi menor do que as variâncias dos preços nos períodos pré e pós cartel (7.0395E-05, 0,019922, 0,013348, respectivamente).

Entretanto, como a autoridade antitruste deve estar interessada em detectar paralelismo de preço e colusão tácita ou secreta, o valor de longo prazo da variância e da média pode ser importante para confirmar estes sinais de comportamento anticompetitivo, como veremos na próxima seção.

\section{Modelando volatilidade de preço em mercados brasileiros de gasolina}

O primeiro passo para verificar a existência de variâncias condicionais nos mercados de gasolina das cidades selecionadas foi a estimação de um processo $\mathrm{AR}(q)$ para o preço e a variância. ${ }^{10}$ Para os três mercados analisados, utilizamos um $\operatorname{AR}(1)^{11}$ para a equação da média do preço. Com relação a São Paulo e Recife, a variável dummy para conspiração mostrou ser estatisticamente significante, de forma que a média do preço da gasolina em São Paulo e em Recife durante colusão foram respectivamente 0,02 e 0,11 centavos por litro maior do que a média do preço no período sem colusão (Tabelas 1 e 3). Ou seja, nós aceitamos a hipótese de maiores preços em São Paulo e em Recife durante o suposto período de cartel. Todavia, nós não encontramos

9 Curiosamente, o preço de cartel mantido em João Pessoa.

$10 \mathrm{O}$ teste de raiz unitária (Augmented Dickey-Fuller test) das séries evidenciou que elas são I(0). Para São Paulo, a regressão com intercepto e tendência e três defasagens o valor calculado foi de -3.9196 e para Florianópolis e Recife a regressão com intercepto e um defasagem o valor calculado foi de -2.658 e -3.5693 respectivamente.

11 Escolhemos a ordem do processo AR usando o critério de Akaike (AIC) e de Swartz (SC). 
um coeficiente estatisticamente significante para a dummy na equação da média para os preços de Florianópolis (Tabela 2).

Tabela 1. Modelando heteroscedasticidade condicional do Mercado de gasolina de São Paulo

\begin{tabular}{|c|c|c|c|c|c|c|c|}
\hline \multicolumn{4}{|c|}{ Equação da média, variável dependente: preço } & \multicolumn{4}{|c|}{$\begin{array}{l}\text { Equação da Variância, variável dependente } \\
\text { variância não condicional }\end{array}$} \\
\hline Regressor & Coef. & St. Error & T-Ratio[Prob] & Regressor & Coef. & St. Error & T-Ratio[Prob] \\
\hline Const & 0.75652 & 0.18557 & $4.072[0.000]$ & Const & 0.00094 & 0.00049 & $1.8948[0.064]$ \\
\hline Price(-1) & 0.68500 & 0.07723 & $8.869[0.000]$ & $\operatorname{Res}(-1)$ & 0.34036 & 0.14508 & $2.3461[0.023]$ \\
\hline Dummy & 0.02311 & 0.0062 & $3.712[0.001]$ & Dummy & -0.0006 & 0.00119 & $-0.563[0.576]$ \\
\hline \multicolumn{8}{|c|}{ Testes de Diagnóstico } \\
\hline & & \multicolumn{2}{|c|}{ Versão LM [Prob] } & & \multicolumn{2}{|c|}{ Versão LM $\quad$ [Prob] } \\
\hline \multicolumn{2}{|c|}{ Correlação Serial } & \multicolumn{2}{|c|}{$\mathrm{CHSQ}(1)=3.8218[0.051]$} & \multicolumn{2}{|c|}{ Correlação Serial } & \multicolumn{2}{|c|}{$\mathrm{CHSQ}(1)=0.5164[0.472]$} \\
\hline \multicolumn{2}{|c|}{ Forma Funcional } & \multicolumn{2}{|c|}{$\mathrm{CHSQ}(1)=6.7619[0.009]$} & \multicolumn{2}{|c|}{ Forma Funcional } & \multicolumn{2}{|c|}{$\operatorname{CHSQ}(1)=0.7329[0.392]$} \\
\hline \multicolumn{2}{|c|}{ Normalidade } & \multicolumn{2}{|c|}{$\mathrm{CHSQ}(2)=52.139[0.000]$} & \multicolumn{2}{|c|}{ Normalidade } & \multicolumn{2}{|c|}{$\mathrm{CHSQ}(2)=663.43[0.000]$} \\
\hline \multicolumn{2}{|c|}{ Heteroscedasticidade } & \multicolumn{2}{|c|}{$\mathrm{CHSQ}(1)=1.3952[0.238]$} & \multicolumn{2}{|c|}{ Heteroscedasticidade } & \multicolumn{2}{|c|}{$\mathrm{CHSQ}(1)=0.2335[0.629]$} \\
\hline
\end{tabular}

Fonte: Elaborado pelos autores.

Tabela 2. Modelando heteroscedasticidade condicional do mercado de gasolina de Florianópolis

\begin{tabular}{|c|c|c|c|c|c|c|c|}
\hline \multicolumn{4}{|c|}{ Equação da média, variável dependente: preço } & \multicolumn{4}{|c|}{$\begin{array}{l}\text { Equação da Variância, variável dependente: } \\
\text { variância não condicional }\end{array}$} \\
\hline Regressor & Coef. & St. Error & T-Ratio[Prob] & Regressor & Coef. & St Error & T-Ratio[Prob] \\
\hline Const & 14.484 & 0.32839 & $4.4106[0.000]$ & Const & 0.04632 & 0.02427 & $1.9085[0.062]$ \\
\hline Price(-1) & 0.4118 & 0.13216 & $3.1162[0.003]$ & $\operatorname{Res}(-1)$ & 0.02869 & 0.14517 & $0.1976[0.884]$ \\
\hline Dummy & 0.0599 & 0.04953 & $1.2105[0.232]$ & Dummy & -0.0355 & 0.03105 & $-1.143[0.258]$ \\
\hline \multicolumn{8}{|c|}{ Testes de Diagnóstico } \\
\hline & & \multicolumn{2}{|c|}{ Versão LM [Prob] } & & \multicolumn{2}{|c|}{ Versão LM } \\
\hline \multicolumn{2}{|c|}{ Correlação Serial } & \multicolumn{2}{|c|}{$\mathrm{CHSQ}(1)=2.3664[0.124]$} & \multicolumn{2}{|c|}{ Correlação Serial } & \multicolumn{2}{|c|}{$\operatorname{CHSQ}(1)=0.0053[0.942]$} \\
\hline \multicolumn{2}{|c|}{ Forma Funcional } & \multicolumn{2}{|c|}{$\mathrm{CHSQ}(1)=3.5617[0.059]$} & \multicolumn{2}{|c|}{ Forma Funcional } & \multicolumn{2}{|c|}{$\mathrm{CHSQ}(1)=0.0006[0.994]$} \\
\hline \multicolumn{2}{|c|}{ Normalidade } & \multicolumn{2}{|c|}{$\mathrm{CHSQ}(2)=479.32[0.000]$} & \multicolumn{2}{|c|}{ Normalidade } & \multicolumn{2}{|c|}{$\mathrm{CHSQ}(2)=2769.8[0.000]$} \\
\hline \multicolumn{2}{|c|}{ Heteroscedasticidade } & \multicolumn{2}{|c|}{$\mathrm{CHSQ}(1)=2.0131[0.156]$} & \multicolumn{2}{|c|}{ Heteroscedasticidade } & \multicolumn{2}{|c|}{$\mathrm{CHSQ}(1)=1.0093[0.315]$} \\
\hline
\end{tabular}

Fonte: Elaborado pelos autores. 
Tabela 3. Modelando heteroscedasticidade condicional do mercado de gasolina de Recife

\begin{tabular}{|c|c|c|c|c|c|c|c|}
\hline \multicolumn{4}{|c|}{ Equação da média, variável dependente: preço } & \multicolumn{4}{|c|}{$\begin{array}{l}\text { Equação da Variância, variável dependente } \\
\text { variância não condicional }\end{array}$} \\
\hline Regressor & Coef. & St. Error & T-Ratio[Prob] & Regressor & Coef. & St Error & T-Ratio[Prob] \\
\hline Const & 18.292 & 0.30906 & $5.9185[0.000]$ & Const & 0.01067 & 0.0027 & $3.9477[0.000]$ \\
\hline Price $(-1)$ & 0.29225 & 0.11930 & $2.4498[0.018]$ & Resq(-1) & 0.11052 & 0.1403 & $0.78751[0435]$ \\
\hline Dummy & 0.11858 & 0.03328 & $3.5628[0.001]$ & Dummy & -0.0994 & 0.0039 & $-2.4866[0016]$ \\
\hline \multicolumn{8}{|c|}{ Teste de Diagnostico } \\
\hline & & \multicolumn{2}{|c|}{$\frac{\text { Versão LM [Prob] }}{2 \text { CHSO(1) }=13666[0242]}$} & \multirow{2}{*}{\multicolumn{2}{|c|}{\begin{tabular}{|l} 
Correlacão Serial \\
\end{tabular}}} & \multicolumn{2}{|c|}{ Versão LM [Prob] } \\
\hline \multicolumn{2}{|c|}{ Correlação Serial } & $\mathrm{CHSQ}(1)$ & $1.3666[0.242]$ & & & $\mathrm{CHSQ}(1)$ & $=2.773[0.096]$ \\
\hline \multicolumn{2}{|c|}{ Forma Funcional } & \multicolumn{2}{|c|}{$\mathrm{CHSQ}(1)=4.1010[0.043]$} & \multicolumn{2}{|c|}{ Forma Funcional } & \multicolumn{2}{|c|}{$\operatorname{CHSQ}(1)=0.066[0.797]$} \\
\hline \multicolumn{2}{|c|}{ Normalidade } & \multicolumn{2}{|c|}{$\mathrm{CHSQ}(2)=1.8780[0.391]$} & \multicolumn{2}{|c|}{ Normalidade } & \multicolumn{2}{|c|}{$\mathrm{CHSQ}(2)=249.6[0.000]$} \\
\hline \multicolumn{2}{|c|}{ Heteroscedasticidade } & \multicolumn{2}{|c|}{$\mathrm{CHSQ}(1)=8.3213[0.004]$} & \multicolumn{2}{|c|}{ Heteroscedasticidade } & \multicolumn{2}{|c|}{$\mathrm{CHSQ}(1)=0.416[0.519]$} \\
\hline
\end{tabular}

Fonte: Elaborado pelos autores.

Os resultados da estimação da equação da variância foram como segue: o sinal da variável dummy mostrou um efeito negativo da ação anticompetitiva sobre a variância. Este resultado está de acordo com a hipótese anterior, mas este coeficiente foi estatisticamente significante apenas para o mercado de Recife (Tabelas 1, 2 e 3).

O segundo passo foi testar o efeito $\mathrm{ARCH}$, isto é, a existência de variância condicional. Em todos os casos, procedemos o ARCH (1) até ARCH (4). Para a série de preços de São Paulo, a versão do Multiplicador de Lagrange do teste produziu uma estatística de 4.9611 em $\mathrm{ARCH}(1),{ }^{12}$ o qual está acima de 95 por cento do valor crítica de $x^{2}{ }_{(1)}$, e consequentemente nós rejeitamos a hipótese de que não existe efeitos ARCH no processo AR (1) das séries de preços. Chega-se à mesma conclusão caso se considere a versão F do teste (Tabela 4).

12 Os mesmos resultados são alcançados se considerarmos $\mathrm{ARCH}$ (2), mas em $\mathrm{ARCH}$ (3) e $\mathrm{ARCH}(4)$ não podemos rejeitar a hipóteses de que não há $\mathrm{ARCH}$. 
Tabela 4. Teste de Heteroscedasticidade Condicional Autoregressiva dos Residuals (caso OLS): preços de São Paulo

\begin{tabular}{|c|c|c|c|c|}
\hline \multicolumn{2}{|l|}{ Variável dependente: Preço } & & & \\
\hline \multicolumn{5}{|c|}{\begin{tabular}{|l} 
Lista das variáveis na regressão: Constant Price(-1) Dummy \\
\end{tabular}} \\
\hline \multicolumn{5}{|c|}{51 observações usadas para a estimação de 2 a 52} \\
\hline & $\mathrm{ARCH}(1)$ & $\mathrm{ARCH}(2)$ & $\mathrm{ARCH}(3)$ & $\mathrm{ARCH}(4)$ \\
\hline $\begin{array}{|ll|}\text { Estatística } & \text { Multiplicador de } \\
\text { Lagrange } & \end{array}$ & $X^{2}(1)=4.9611$ & $X^{2}(2)=5.6959$ & $X^{2}(3)=5.5894$ & $X^{2}(3)=5.6699$ \\
\hline Estatística F & $\begin{array}{r}F(1,47)=5.0647 \\
{[0.029]}\end{array}$ & $\begin{array}{c}\mathrm{F}(2,46)=2.8917 \\
{[0.066]}\end{array}$ & $\begin{array}{r}F(3,45)=1.8463 \\
{[0.152]}\end{array}$ & $\begin{array}{r}F(4,44)=1.3758 \\
{[0.258]}\end{array}$ \\
\hline
\end{tabular}

Fonte: Elaborado pelos autores.

Todavia, a rejeição da hipótese de que não existem efeitos $\mathrm{ARCH}$ não necessariamente implica que a variância condicional do processo AR é variável, pois devemos verificar se os resíduos do processo AR são serialmente não correlacionados. Nós realizamos o teste de correlação serial nos resíduos e sua versão LM gerou a rejeição da hipótese de que os resíduos são serialmente não correlacionados ${ }^{13}$, Este resultado significa que a existência do efeito $\mathrm{ARCH}$ nos preços da gasolina de São Paulo não é conclusiva. Em outras palavras, dado o padrão da variância dos preços, não podemos confirmar uma menor volatilidade nos preços.

Considerando agora as séries de preços de gasolina de Florianópolis, não podemos rejeitar a hipótese de que não existe heteroscedasticidade condicional autoregressiva nos resíduos em ambas estatísticas, LM e F (Tabela 5).

Tabela 5. Teste de Heteroscedasticidade Condicional Autoregressivados Resíduos (caso OLS): preço de Florianópolis

\begin{tabular}{|c|c|c|c|c|}
\hline \multicolumn{5}{|l|}{ Variável dependente: Preço } \\
\hline \multicolumn{5}{|c|}{ Lista das variáveis na regressão: Constant Price(-1) Dummy } \\
\hline \multicolumn{5}{|c|}{51 observações usadas para a estimação de 2 a 52} \\
\hline & $\mathrm{ARCH}(1)$ & $\mathrm{ARCH}(2)$ & $\mathrm{ARCH}(3)$ & $\mathrm{ARCH}(4)$ \\
\hline \begin{tabular}{|l|} 
Estatística Multiplicador de \\
Lagrange
\end{tabular} & $\begin{array}{r}X^{2}(1)=0.13776 \\
{[0.771]}\end{array}$ & $\begin{array}{r}X^{2}(2)=0.13021 \\
{[0.937]}\end{array}$ & $\begin{array}{r}X^{2}(3)=0.1401 \\
{[0.987]}\end{array}$ & $\begin{aligned} X^{2}(4)= & 0.18872 \\
& {[0.996] }\end{aligned}$ \\
\hline Estatística F & $\begin{array}{r}\mathrm{F}(1,47)=0.12730 \\
{[0.723]}\end{array}$ & $\begin{array}{r}\mathrm{F}(2,46)=0.0588 \\
{[0.943]}\end{array}$ & $\begin{array}{r}\mathrm{F}(1,47)=0.0413 \\
{[0.989]}\end{array}$ & $\begin{array}{r}\mathrm{F}(4,44)=0.0408 \\
{[0.997]}\end{array}$ \\
\hline
\end{tabular}

Fonte: Elaborado pelos autores.

13 Teste da correlação serial dos resíduos (OLS) para os preços de São Paulo: Estatística Lagrange Multiplier $x^{2}{ }_{(1)}=3.8218$ e valor p igual a 0.051 . 
Com relação a série de preços de Recife, a versão do Multiplicador de Lagrange do teste produziu uma estatística de 2.9032 em $\mathrm{ARCH}(1),{ }^{14}$ o qual está acima de 90 por cento do valor crítica de $\mathfrak{X}^{2}{ }_{(1)}$, e consequentemente nós rejeitamos a hipótese de que não existe efeitos $\mathrm{ARCH}$ no processo AR (1) das séries de preços. Chega-se à mesma conclusão caso se considere a versão $F$ do teste (Tabela 6). No teste de correlação serial nos resíduos, em sua versão LM, gerou a não rejeição da hipótese de que os resíduos são serialmente não correlacionados. ${ }^{15}$ Este resultado significa que a existência do efeito $\mathrm{ARCH}(1)$ nos preços da gasolina de Recife é conclusiva. ${ }^{16}$ Em outras palavras, dado o padrão da variância dos preços, podemos confirmar uma menor volatilidade nos preços.

Tabela 6. Teste de Heteroscedasticidade Condicional Autoregressiva dos Residuos (caso OLS): preços de Recife

\begin{tabular}{|c|c|c|c|c|}
\hline \multicolumn{5}{|l|}{ Variável dependente: Preço } \\
\hline \multicolumn{5}{|c|}{ Lista das variáveis na regressão: Constant Price(-1) Dummy } \\
\hline \multicolumn{5}{|c|}{51 observações usadas para a estimação de 2 a 52} \\
\hline & $\mathrm{ARCH}(1)$ & $\mathrm{ARCH}(2)$ & $\mathrm{ARCH}(3)$ & $\mathrm{ARCH}(4)$ \\
\hline $\begin{array}{|ll|}\text { Estatística } & \text { Multiplicador de } \\
\text { Lagrange } & \\
\end{array}$ & $\begin{array}{r}X^{2}(1)=2.9032 \\
{[0.088]}\end{array}$ & $\begin{array}{r}X^{2}(2)=2.6722 \\
{[0.263]}\end{array}$ & $\begin{array}{r}X^{2}(3)=2.4973 \\
{[0.476]}\end{array}$ & $\begin{array}{r}X^{2}(4)=2.7163 \\
{[0.606]}\end{array}$ \\
\hline Estatística F & $\begin{array}{r}\mathrm{F}(1,48)=2.8384 \\
{[0.099]}\end{array}$ & $\begin{array}{r}\mathrm{F}(2,47)=1.2731 \\
{[0.289]}\end{array}$ & $\begin{array}{r}F(3,46)=0.7735 \\
{[0.515]}\end{array}$ & $\begin{array}{r}F(4,45)=0.6200 \\
{[0.651]}\end{array}$ \\
\hline
\end{tabular}

Fonte: Elaborado pelos autores.

\section{Considerações finais}

O objetivo central deste trabalho foi apresentar uma metodologia complementar para detectar colusão em mercados de gasolina a varejo. Seguindo a literatura, nós realizamos um exercício para mostrar como os modelos ARCH e GARCH podem ser usados para examinar o comportamento da média e da variância da distribuição de preço.

Portanto acreditamos ter contribuído com a análise do problema em duas direções: primeiro, a técnica econométrica que pode ser usada para testar a presença de comportamento colusivo, em mercados onde colusão é provável de ocorrer, demanda somente dados

14 Resultado contrário é alcançado quando se considera os $\mathrm{ARCH}$ (2), $\mathrm{ARCH}$ (3) e $\mathrm{ARCH}$ (4). Isto é, não podemos rejeitar a hipóteses de que não há $\mathrm{ARCH}$.

15 Teste da correlação serial dos resíduos (OLS) para os preços de Recife: Estatística Lagrange Multiplier $x^{2}(1)=1.3666$ e valor $p$ igual a 0.242 .

16 Neste caso, não se conseguiu especificar um modelo $\mathrm{GARCH}$ diferente de $\operatorname{GARCH}(0,1)$. 
de preços médios; segundo, estes modelos requerem uma quantidade mínima de dados (séries temporais de preço de mercados cartelizados antes, durante e depois de um período conhecido ou hipotético de conspiração).

Finalmente, uma sugestão para trabalhos futuros é incluir dados de mercados de mais cidades brasileiras, bem como comparar os resultados do procedimento oficial com os da metodologia proposta aqui.

\section{Referências Bibliograficas}

ANP. Preços de combustíveis. Disponível em: < http://www.anp.br > . Acesso em: jan/dez 2006.

BAKER, J. Two Sherman Act section 1 dilemmas: parallel pricing, the oligopoly problem, and contemporary economic theory. The Antitrust Bulletin. Spring. 1993.

BOLOTOVA, Y.; CONNOR, J.M.; MILLER, D.J. The impact of collusion on price behavior: empirical results from recent cases. The Third Annual International Organization Conference. Atlanta, Georgia, April 2005.

BUCCIROSSI, P. Does paralell behavior provide some evidence of collusion? Working Paper. Roma: Lear. 2002. Disposable in: <http://www.lear-sas.it/frameset.cfm>.

COMPETITION BUREAU. Application of the Competition Act to the current fluctuations in gasoline prices. [s.l.]: [s.n.]. October. 1994. Disponível em: <http://www.cb-bc.gc.ca/ epic/internet/incb-bc.nsf/vwGeneratedInterE/ct01549e.html > . Acesso em: 15/12/2003.

ENDERS, W. Applied econometric time series. New York: John Wiley 8 Sons, Inc. 1996.

GROSSMANN, P.Z. What do we mean by cartel success? In: GROSSMAN, P.Z. (Ed.) How cartels endure and how they fail: studies of industrial collusion. Cheltenham, UK: Edward Elgar, 2004.

KOVÁC, E.; PUTZOVÁ, A.; ZEMPLINEROVÁ, A. A survey of collusion in gasoline markets. Discussion Paper Series, n. 2005 - 148. Charles University, Center for Economic Research and Graduate Education, Academy of Siences of the Czech Republic, Economics Institute. 2005

KOVACIC, W. The identification and proof of horizontal agreements under the antitrust laws. The Antitrust Bulletin. Spring. 1993.

MACLEOD, W.B. A theory of conscious parallelism. European Economic Review, v. 27, p. 25-44, 1985.

NORMANN, H-T. Conscious parallelism in asymmetric oligopoly. Metroeconomica, v. 51, n. 3, p. 43-366, 2000.

OECD. Global Forum on Competition: roundtable on prosecuting cartels without direct evidence of agreement (contribution from Brazil), 2006. Disposable in: < http:// www.oecd.org/dataoecd/61/28/36063750.pdf > .

PHLIPS, L. Collusion and predation: on the detection of collusion and predation. In: . Applied industrial economics. Cambridge: Cambridge University Press. 1998

RAGAZZO, C.E.J.; SILVA, R.M. Aspectos econômicos e jurídicos sobre cartéis na revenda de combustíveis: uma agenda para investigações. Documento de Trabalho n. 40. Brasília: SEAE. 2006 
SLADE, M.E. Interfirm rivalry in a repeated game: an empirical test of tacit collusion. The Journal of Industrial Economics, v. 35, n. 4, 1987. p. 499-516.

VASCONCELOS, S.P.; VASCONCELOS, C.R.F. Investigações e obtenção de provas de cartel: porque e como observar paralelismo de conduta. Ensaios FEE, v. 26, n. 2. 2005 YAO, D.; DeSANTI, S.S. Game theory and the legal analysis of tacit collusion. The Antitrust Bulletin. Spring, 1993. 